\title{
Einführung - Neutralität und Entwicklungspolitik
}

\section{Catherine Schümperli Younossian}

\section{OpenEdition}

\section{Journals}

Electronic version

URL: http://journals.openedition.org/sjep/64

DOI: $10.4000 /$ sjep. 64

ISSN: 1663-9677

\section{Publisher}

Institut de hautes études internationales et du développement

\section{Printed version}

Date of publication: 1 avril 2008

Number of pages: XI-XVII

ISBN: 978-2-940415-02-1

ISSN: $1660-5926$

\section{Electronic reference}

Catherine Schümperli Younossian, «Einführung - Neutralität und Entwicklungspolitik »,

Schweizerisches Jahrbuch für Entwicklungspolitik [Online], 27-1 | 2008, Online erschienen am: 19

November 2009, abgerufen am 22 September 2020. URL : http://journals.openedition.org/sjep/64

DOI : https://doi.org/10.4000/sjep.64 


\section{Einführung}

\section{Neutralität und Entwicklungspolitik}

W

ELCHEN WERT besitzt heutzutage noch die „Neutralität“, Gründungsmythos der schweizerischen nationalen Identität? Das Neutralitätskonzept war im Laufe des 20. Jahrhunderts Gegenstand verschiedener Auslegungen und steht zurzeit im Mittelpunkt der aussenpolitischen Debatte der Schweiz. Im Sommer 2007 brachte die Zeitung Le Temps ein Dossier zur Neutralität heraus, in dem vermerkt wird, dass die Neutralität heute merkwürdigerweise wieder in Mode kommt, wobei die sie rechtfertigenden Bedrohungen aber nicht mehr bestehen ${ }^{1}$. Die aktuelle Debatte dreht sich um zwei mögliche Auslegungen des Neutralitätskonzepts, und zwar um ,aktive“ oder „,integrale“ Neutralität. Daneben ist aber auch die etwas missbräuchliche Verwendung des Begriffs „Klimaneutralität“ zu erwähnen, der am Rande der Debatte über den Klimawandel angewandt wird. Nachstehend befassen wir uns mit einigen Schwerpunkten der Neutralitätsdebatte im Jahr 2007, die in verschiedenen Kapiteln des vorliegenden Jahrbuchs ausführlicher behandelt werden.

\section{Der Begriff „klimaneutrale Schweiz“}

Überraschend ist die Bezugnahme auf das Konzept einer „klimaneutralen Schweiz", das vom Staatssekretariat für Wirtschaft (SECO) vorgeschlagen wird. Laut dem SECO besteht das Konzept darin, „dass sich alle Verursacher von Treibhausgasemissionen in der Schweiz verpflichten, im Ausmass ihrer Emissionen Kompensationsmassnahmen im Ausland zu leisten“2. Dabei handelt es sich für die Schweiz um eine ,geschickte“ Art und Weise, sich zu einer Reduktion ihrer Treibhausgasemissionen um hundert Prozent zu verpflichten, ohne sich verbindliche Ziele setzen zu müssen! Die Reaktionen darauf waren prompt und undifferenziert: Während der Vorschlag von den Wirtschaftskreisen begrüsst wurde, die darin ein Mittel zur Vermeidung von allzu restriktiven Massnahmen sehen, wurde er von den nichtstaatlichen Umwelt- und Entwicklungsorganisationen als „scheinbar gute Idee“, die entwicklungspolitisch unvertretbar sei, scharf abgelehnt. Das Konzept der „Klimaneutralität“" sei nicht universal, da es nur einigen Staaten erlaube, sich einer effektiven Reduzierung der $\mathrm{CO}_{2}$-Emissionen zu entziehen. Der Markt der Kohlenstoffkredite sei ungerecht, da die armen Länder kaum davon profitieren, weil sie nur sehr wenig $\mathrm{CO}_{2}$ pro Einwohner ausstossen. Schliesslich gestatte es das Konzept der Schweiz, den sich aus dem „Verursacherprinzip“ und der ,gemeinsamen, aber differenzierten Verantwortung“33 angesichts der Klimaerwärmung ergebenden Verpflichtungen

1 „Que vaut encore la neutralité?“،, Le Temps, 3. Juli 2007. Zur Herausgabe des Buches von J.-J. Langendorf, Histoire de la neutralité. Une perspective, Gollion, Infolio, 2007.

2 T. Roth, „Klimaneutrale Schweiz - eine Option für die schweizerische Klimapolitik nach 2012?“", Die Volkswirtschaft, Nr. 9, 2007. 
mit geringem Aufwand nachzukommen, bzw. diese Verpflichtungen geschickt zu umgehen. (Kapitel 10, „Umwelt und Entwicklung“).

Lassen wir diesen ungerechtfertigten Gebrauch des Begriffs „Neutralität“ hiermit beiseite und wenden wir uns einer herkömmlicheren Definition der „Neutralität“ im Rahmen der Aussen- und Sicherheitspolitik der Schweiz zu. „Neutralität“ im Sinne des Völkerrechts bedeutet „militärische Nichtbeteiligung an einem bewaffneten Konflikt zwischen anderen Staaten"4. Untersuchen wir die verschiedenen Interpretationen, die in der aktuellen öffentlichen Debatte gebräuchlich sind, bei der sich zwei Visionen der Neutralität gegenüberstehen: diejenige der „aktiven“ Neutralität, die von der Vorsteherin des Departements für auswärtige Angelegenheiten, Bundesrätin Micheline Calmy-Rey, gefördert wird, und diejenige der „,integralen“ Neutralität, die von der konservativen Bewegung AUNS (Aktion für eine unabhängige und neutrale Schweiz) vertreten wird.

\section{„Aktive“ Neutralität versus „integrale“ Neutralität}

Das Konzept der Neutralität ist Kernpunkt der Aussenpolitik von Bundesrätin Calmy-Rey; sie ist das Instrument, dem sie neuen Auftrieb verleihen will. Mit ihrem Eintreten für eine so genannte ,aktive“ Neutralität versucht die Bundesrätin, ein Tabu aufzuheben, indem sie für die Schweiz die Möglichkeit geltend macht, einen Sitz im UN-Sicherheitsrat zu fordern. Der Bericht zur Aussenpolitik 2007 bietet diesbezüglich eine detaillierte Argumentation, wobei er anführt, dass die Beteiligung der Schweiz an den Arbeiten des Sicherheitsrates die Bedeutung unterstreichen könnte, welche die Schweiz der Transparenz und der Legitimität dieses Organs beimisst. Ferner wird darauf verwiesen, dass andere neutrale Staaten, darunter Österreich, Schweden, Finnland und Irland, einen Sitz im Sicherheitsrat eingenommen haben ${ }^{5}$.

Die Reaktion der AUNS hatte sich bereits seit einiger Zeit angebahnt. Sie wurde in Form eines Argumentariums veröffentlicht, das bezüglich der Relevanz eines Verzichts auf die schweizerische Neutralität eingangs die Frage aufwirft: „unser erfolgreiches Instrument für Sicherheit, Friedensdiplomatie und humanitäre Hilfe ad acta legen ?". In ihrem Schwarzbuch der schweizerischen Neutralität ${ }^{6}$ stellt die AUNS ihre Interpretation der „,integralen Neutralität“ dem Begriff der „,aktiven Neutralität" gegenüber, dessen Abschaffung sie befürwortet. Sie listet ferner die „Neutralitätsverstösse“ auf, die das Departement für auswärtige Angelegenheiten (EDA) sowie das Departement für Verteidigung, Bevölkerungsschutz und Sport (VBS) und das Parlament in ihren Augen begangen haben (Kapitel 1, „Aussenpolitik").

3 M. Egger, „En matière de climat, ,neutralité “ ne rime pas forcément avec ,responsabilité““, Le Temps, 14. Dezember 2007.

4 Eidgenössisches Departement für auswärtige Angelegenheiten (EDA), Das Wichtigste zur Schweizer Neutralität (undatiert), <http://www.eda.admin.ch> > Themen >Völkerrecht >Allgemeines Völkerrecht $>$ Neutralität der Schweiz.

5 Bundesrat, Neutralität (BB1 2007 5531), Anhang 1 zum Aussenpolitischen Bericht, Juni 2007 vom 15. Juni 2007 (BB1 2007 5557).

6 AUNS, Schwarzbuch der schweizerischen Neutralität, Standortbestimmung und Forderungen der AUNS nach 5 Jahren UNO-Mitgliedschaft, Stand: 14. Dezember 2007, <http://www.auns.ch>. 
Die Begriffe „Frieden“ und „Sicherheit“ sind zum Bestandteil internationaler Zusammenarbeit geworden. Sie haben auch die Schweizer Entwicklungszusammenarbeit gezwungen, latente oder offene Konfliktsituationen in ihren Einsatzländern besser zu berücksichtigen ${ }^{7}$. Durch die Annahme des Berichts des Bundesrates über die Sicherheitspolitik im Jahr 1999 wurde eine weiterer Schritt getan. Im Bericht wird der Sicherheitsbegriff auf nichtmilitärische Bedrohungen ausgedehnt, und vor allem wird Schweizer Soldaten die grundsätzliche Möglichkeit geboten, sich an der sicherheitspolitischen Zusammenarbeit in Europa zu beteiligen $^{8}$. In diesem Zusammenhang sind die Teilnahme der Schweiz an der Partnerschaft für den Frieden seit 1996 und der Einsatz von Schweizer Armeeeinheiten im Rahmen internationaler Friedensmissionen unter UN-Mandat (Bosnien-Herzegowina seit 1996, Kosovo seit 1999, Afghanistan seit 2003) zu erwähnen. Die Prinzipien der Neutralität und Solidarität werden darin entsprechend der neuen internationalen Realität neu konzipiert. (Kapitel 11, „Friedens- und Sicherheitspolitik").

„Ohne Sicherheit keine Entwicklung“: Deshalb räumt der Index für das Entwicklungsengagement (Commitment to Development Index, CDI) ${ }^{9}$, der das Engagement der Industrieländer zugunsten der Entwicklung messen soll, der Sicherheit eine bedeutende Stellung ein. Im Jahr 2007 wurde die Schweiz unter den 21 Ländern vom 13. auf den 17. Rang zurückgestuft. Dieses schlechte Resultat erklärt sich insbesondere aus dem sehr geringen Schweizer Beitrag zu den internationalen Friedenserhaltungsoperationen der UNO. (Kapitel 2, „Zusammenarbeit mit den Entwicklungsländern“).

\section{Neutralität und multilaterale Entwicklungszusammenarbeit}

Das Neutralitätsprinzip dient auch anderen Interessen als dem nationalen Sicherheitsinteresse. Die multilaterale Zusammenarbeit, ein wichtiger Bereich der Entwicklungszusammenarbeit, fand in den 90er Jahren nach dem Beitritt der Schweiz zu den hauptsächlichen internationalen Entwicklungsorganisationen neuen Auftrieb. 1992 hat das Volk per Volksabstimmung den Beitritt der Schweiz zum Internationalen Währungsfonds und zur Weltbank mit 55,8 Prozent der Stimmen angenommen und 2002 den Beitritt zur Organisation der Vereinten Nationen mit 54,6 Prozent der Stimmen gutgeheissen. Fast ein halbes Jahrhundert musste vergehen, bis eine kleine Mehrheit des Schweizer Volkes keinen wesentlichen Widerspruch mehr zwischen dem Neutralitätsprinzip und einer

7 Frieden und Sicherheit: Herausforderungen für die internationale Zusammenarbeit, Dossier des Schweizerischen Jahrbuchs für Entwicklungspolitik, Genf, Institut universitaire d'études du développement, 2006.

8 Sicherheit durch Kooperation. Bericht des Bundesrates über die Sicherheitspolitik der Schweiz (SIPOL B 2000) vom 7. Juni 1999 (BB1 1999 7657).

9 Mit dem Index zur Messung des Entwicklungsengagements (Commitment to Development Index, CDI) werden 21 der reichsten Länder nach ihrem Engagement in Bezug auf die Politik zugunsten der armen Länder eingestuft. Der CDI geht über die Grundvergleiche zur öffentlichen Entwicklungshilfe hinaus, indem er die Leistung der Länder anhand von sieben für die Entwicklungsländer wichtigen Kriterien misst: internationale Entwicklungshilfe, Handel, Investitionen, Migrationspolitik, Umwelt, Sicherheit, Technologie (siehe <http://www.cgdev.org/cdi >). 
vollwertigen politischen Beteiligung an den internationalen Organisationen sah und diese Beteiligung wird von einem Teil der schweizerischen öffentlichen Meinung wie auch von der AUNS im Namen der Neutralität weiterhin bekämpft ${ }^{10}$.

Im Laufe des 20. Jahrhunderts hatte die Schweizer Neutralitätspolitik verschiedene Aspekte angenommen. Während des Zweiten Weltkriegs und des Ost-WestKonflikts verfolgte die Schweiz eine so genannte ,integrale“ Neutralitätspolitik, die durch keinerlei militärische, wirtschaftliche oder politische Einmischung in Konflikte zwischen anderen Staaten gekennzeichnet war ${ }^{11}$. Nach der Beendigung der bipolaren Weltordnung ergab sich für die Schweiz eine neue Situation. Wie alle neutralen Staaten hat sie sich bereit erklärt, nichtmilitärische Zwangsmassnahmen, die von der internationalen Staatengemeinschaft zur Aufrechterhaltung oder Wiederherstellung des Friedens ergriffen werden, als Zeichen der Solidarität zu unterstützen. Seit 1990 nimmt die Schweiz regelmässig an internationalen Sanktionen teil. Wie wird die Neutralität in den kommenden Jahren wahrgenommen und ausgelegt werden? Diese Frage wird im Zentrum der aussenpolitischen Debatten stehen, die sich als lebhaft und polemisch ankündigen.

Das EDA, das sich der Bedeutung der Wahrnehmung der schweizerischen öffentlichen Meinung bewusst ist, hat eine breit angelegte Informationskampagne für die Bürgerinnen und Bürger zu sechs Schwerpunktthemen seiner Aussenpolitik lanciert, unter denen die Neutralität als erster Punkt aufgeführt wird $^{12}$. In einem originellen und innovativen Ansatz - wenngleich man an seiner Wirkung und am Repräsentationswert der Meinungsumfrage zweifeln mag werden die Bürgerinnen und Bürger aufgefordert, ihre Meinung zu den wesentlichen Orientierungen der Schweizer Aussenpolitik zu äussern, indem sie dem EDA einen Fragenbogen ausgefüllt zurückschicken. Etwa acht Monate nach Beginn der Kampagne sind über tausend Antworten eingegangen. Man erfährt darin insbesondere, dass 63 Prozent der Befragten die Neutralität als sehr wichtig erachten. Wird von einem Drittel der Antworten betont, die jetzige Neutralitätspraxis sei zu defensiv, so wird die Neutralitätspolitik von der Hälfte der Antworten als zufriedenstellend beurteilt ${ }^{13}$. Die AUNS hat ihrerseits eine Volksinitiative: „Für die Stärkung der Volksrechte in der Aussenpolitk (Staatsverträge vors Volk!)“ lanciert, die von der Bundeskanzlei im Februar 2008 für gültig erklärt wurde ${ }^{14}$. Die AUNS hat bis September 2009 Zeit, um die erforderlichen 100000 Unterschriften zu sammeln.

Die Neutralität ist unbestreitbar ein wichtiges Identitätsmerkmal der Schweizer

10 C. Schümperli, La politique suisse de solidarité internationale. De la coopération au développement global, coll. Le Savoir suisse, Lausanne, Presses Polytechniques et Universitaires Romandes (PPUR), 2007.

11 L. Goetschel, M. Bernath und D. Schwarz, Schweizerische Aussenpolitik: Grundlagen und Möglichkeiten, Zürich, NZZ-Verlag, 2002.

12 EDA, Die Aussenpolitik im Dialog, Bern (nicht datiert).

13 EDA, <http://www.eda.admin.ch> > Dokumentation > Publikationen > Die Aussenpolitik im Dialog (Einsichtnahme: Februar 2008).

14 Bundeskanzlei, Eidgenössische Volksinitiative: „Für die Stärkung der Volksrechte in der Aussenpolitk (Staatsverträge vors Volk!)“ (BB1 2008 1485). 
Aussenpolitik. Zwar hat sich das Konzept in der jüngeren Geschichte der Schweiz weiterentwickelt und konnte sich den neuen internationalen Realitäten anpassen, doch ist auf ihr grundlegendes Ziel zu verweisen: „Die Neutralität ist nicht nur ein Sicherheitsinstrument, sondern auch eine Friedensbotschaft. Ein neutraler Staat will vor allem eins: den Krieg verhindern"15. Der Ablauf des Weltgeschehens und die Positionierung der Schweiz auf internationaler Ebene machen eine ständige Neuauslegung der Neutralität erforderlich. Damit sich die Neutralität in einem innenpolitischen Klima, in dem die konservativen Kräfte für eine restriktive Interpretation eintreten, entwickeln kann, ist es wichtig, die Anwendungsfelder der Neutralität klar festzulegen.

$\mathrm{Zu}$ Beginn dieses dritten Jahrtausends ist der Klimawandel zweifelsohne die stärkste Bedrohung für das planetare Gleichgewicht; die diesbezüglich anfallenden Entscheidungen sind zu schwerwiegend und global, um mit einer angeblichen „Klimaneutralität“" herumzuspielen. Zum anderen stehen im Jahr 2008 grosse Debatten auf der Agenda der internationalen Entwicklungszusammenarbeit: Die Frage der Wirksamkeit der Hilfe wird im September in Akkra untersucht und das Thema der Entwicklungsfinanzierung im November in Doha diskutiert. In diesem Kontext wird der Beitrag der Schweiz wesentlich mehr an ihrem solidarischen Engagement als an ihrer Neutralität gemessen werden!

$$
* * *
$$

Migration und Entwicklung: Dossier des Jahrbuchs 2008

Die internationalen Migrationen sind zu einem wesentlichen Thema globaler Tragweite geworden und fortan von Entwicklungsfragen nicht mehr zu trennen. Mit der Lancierung der Initiative von Bern im Jahr 2001 hat die Schweiz der Stärkung der internatioalen Zusammenarbeit im Migrationsbereich einen wichtigen Impuls gegeben, der zur Gründung der Global Commission on International Migration führte.

Weltweit zählt man rund 200 Millionen Migranten, davon sind fast die Hälfte Frauen. In der Schweiz ist eine(r) von drei Einwohnerinnen und Einwohnern ausländischer Herkunft und ein Viertel der erwerbstätigen Bevölkerung besitzt keinen Schweizer Pass. Angesichts der zunehmenden Komplexität der Migrationsströme erscheinen einseitige Ansätze zur Migrationsregulierung immer ineffizienter. Auch wenn die Staaten weiterhin eifersüchtig an ihrer Souveranität im Migrationsbereich festhalten, so werden sie sich nach und nach der Tatsache bewusst, dass nur eine auf internationaler Ebene abgestimmte Aktion zu konstruktiven Lösungen führen kann, die sowohl für die Herkunfts-, Transit- und Bestimmungsländer - die manchmal mehrere dieser Funktionen gleichzeitig ausüben - als auch für die Migrantinnen und Migranten annehmbar sind.

Zusammen mit diesen politischen Initiativen, die ihrerseits zu zahlreichen regionalen und nationalen Befragungen Anlass gaben, erscheinen immer mehr Studien zum Zusammenhang zwischen Migration und Entwicklung (,MigrationDevelopment Nexus"), wenngleich diese Arbeiten derzeit noch weitgehend

15 EDA, Die Aussenpolitik im Dialog, op. cit. 
unzureichend sind, um alle Aspekte des Zusammenhangs zwischen den beiden Phänomenen zu klären. Zum einen werden die Folgen der Migrationsbewegungen auf die Herkunfts- und Aufnahmeländer besser berücksichtigt; zum anderen werden die - lange Zeit sehr kontrovers diskutierten - Auswirkungen der Entwicklung auf die Süd-Nord-Migrationen in ihrer ganzen Vielschichtigkeit erfasst. Wenn die humanitäre Hilfe die - namentlich regionale - Zwangsauswanderung auch im ersten Augenblick verhüten kann, so verstärkt die wirtschaftliche Entwicklung den Migrationsdruck jedoch zuweilen auf mittlere Sicht, bevor sie längerfristig zu dessen Rückgang beiträgt (Migration Hump). Somit mochten die Visionen der Entwicklungszusammenarbeit und der Migrationspolitik in der Vergangenheit manchmal als gegensätzlich erscheinen und sind es in einer auf die unmittelbare Zukunft ausgerichteten Perspektive auch weiterhin. Hingegen lassen sich diese unterschiedlichen Sichtweisen aneinander anpassen, wenn man einen nachhaltigen Entwicklungsansatz verfolgt. Diese verschiedenen Visionen werden im nächsten Dossier des Jahrbuchs (Herausgabe Winter 2008-2009) analysiert und erörtert werden ${ }^{16}$.

$$
* * *
$$

Ab diesem Jahr wird das Schweizerische Jahrbuch für Entwicklungspolitik neu vom Genfer Institut de hautes études internationales et du développement veröffentlicht, das aus der Zusammenlegung des Institut universitaire d'études du développement (IUED), früherer Herausgeber, und des Institut universitaire de hautes études internationales (HEI) am 1. Januar 2008 hervorgegangen ist.

Als weitere Neuheit werden ab 1. Juni 2008 sämtliche Texte der Jahrbücher der letzten fünf Jahre (2002-2007) im Internet frei zugänglich gemacht. Auch soll ein grösserer Zusammenhang zwischen der Papierversion des Jahrbuchs und der Website gewährleistet werden. Zum Beispiel werden auf unseren Internetseiten zusätzliche statistische Daten angeboten ${ }^{17}$.

Das Redaktionskomitee dankt den Autorinnen und Autoren der in diesem Band Schweizerisches Jahrbuch für Entwicklungspolitik veröffentlichten Texte, wie auch den zahlreichen Spezialisten der Bundesverwaltung, der EntwicklungsNRO und aus Akademikerkreisen, die unserer Publikation durch ihre kritische Überprüfung und ihre wertvollen Ratschläge zu grosser Seriosität verhelfen. Unser Dank richtet sich ebenfalls an all jene, die zur Gestaltung, Realisierung und Übersetzung des Jahrbuchs beigetragen haben. Auf ihrer Kompetenz und ihrem Engagement beruht die Zuverlässigkeit dieses Nachschlagewerks. Wir danken auch der Direktion für Entwicklung und Zusammenarbeit (DEZA) für ihre finanzielle Unterstützung.

Zum Abschluss möchte das Redaktionskomitee Jacques Vallet, Redaktionssekretär des Jahrbuchs seit seiner Einführung, und Xavier Tschumi Canosa, Jahrbuchredaktor, seine Anerkennung und Würdigung aussprechen. Jacques Vallet hat fast dreissig Jahre lang mit grosser Kompetenz und Engagement zur Veröffentlichung der zahlreichen Bände der Jahrbuchreihe beigetragen. Er hat

16 D. Efionayi, Auszug aus der Übersicht zum Dossier „Migration und Entwicklung“, Oktober 2007.

17 Siehe die Internetseite zum Jahrbuch auf der Website des Institut de hautes études internationales et du développement: <http://graduateinstitute.ch/development/publications/aspd.html>. 
an nahezu 300 Sitzungen des Redaktionskomitees teilgenommen, den Herstellungsprozess des Jahrbuchs von der Gestaltung bis zum Druck begleitet und diesem gleichzeitig einen breiten Vertrieb gesichert. Jacques Vallet hat nunmehr das Ruhestandsalter erreicht und sein Amt Anfang Januar 2008 aufgegeben. Seine menschliche und warmherzige Persönlichkeit wird das gesamte Jahrbuchteam künftig missen. Xavier Tschumi Canosa hat fast sechs Jahre lang zur Redaktion der Jahrbuchtexte beigetragen und war für den Band über Friedens- und Sicherheitspolitk (Dossier 2006) verantwortlich. Seine Arbeit war durch Präzision und Seriosität gekennzeichnet, seine Beziehungen zu den Kollegen von menschlicher Wärme und Freundschaft geprägt. Xavier Tschumi Canosa interessiert sich sehr für Umweltfragen und wird seine Laufbahn als wissenschaftlicher Mitarbeiter beim Bundesamt für Umwelt fortsetzen. Das Redaktionskomitee dankt Jacques Vallet und Xavier Tschumi Canosa herzlich für die Jahre ihres Engagements und wünscht ihnen alles Gute für den neuen Lebensabschnitt.

Catherine Schümperli Younossian

Redaktionsleiterin 